\title{
Characterization of Mutant Myosins of Dictyostelium discoideum Equivalent to Human Familial Hypertrophic Cardiomyopathy Mutants Molecular Force Level of Mutant Myosins May Have a Prognostic Implication
}

\author{
Hideo Fujita, ${ }^{*}$ Seiryo Sugiura,${ }^{*}$ Shin-ichi Momomura, ${ }^{*}$ Masao Omata, ${ }^{*}$ Haruo Sugi, ${ }^{\star}$ and Kazuo Sutoh ${ }^{\S}$ \\ *Second Department of Internal Medicine, University of Tokyo, Hongo, Tokyo 113, Japan; ${ }^{\ddagger}$ Department of Physiology, School of \\ Medicine, Teikyo University, Kaga, Tokyo 173, Japan; and ${ }^{\S}$ Department of Life Sciences, Graduate School of Arts and Sciences, \\ University of Tokyo, Komaba, Tokyo 153, Japan
}

\begin{abstract}
Recent studies have revealed that familial hypertrophic cardiomyopathy (FHC) is caused by missence mutations in myosin heavy chain or other sarcomeric proteins. To investigate the functional impact of FHC mutations in myosin heavy chain, mutants of Dictyostelium discoideum myosin II equivalent to human FHC mutations were generated by site-directed mutagenesis, and their motor function was characterized at the molecular level. These mutants, i.e., R397Q, F506C, G575R, A699R, K703Q, and K703W are respectively equivalent to R403Q, F513C, G584R, G716R, R719Q, and R719W FHC mutants. We measured the force generated by these myosin mutants as well as the sliding velocity and the actin-activated ATPase activity. These measurements showed that the A699R, K703Q, and K703W myosins exhibited unexpectedly weak affinity with actin and the lowest level of force, though their ATPase activity remained rather high. F506C mutant which has been reported to have benign prognosis exhibited the least impairment of the motile and enzymatic activities. The motor functions of R397Q and G575R myosins were classified as intermediate. These results suggest that the force level of mutant myosin molecule may be one of the key factors for pathogenesis which affect the prognosis of human FHC. $(J$. Clin. Invest. 1997. 99:1010-1015.) Key words: hypertrophic cardiomyopathy - myosin heavy chain • recombinant proteins $\bullet$ dictyostelium $\bullet$ site-directed mutagenesis
\end{abstract}

\section{Introduction}

Familial hypertrophic cardiomyopathy $(\mathrm{FHC})^{1}$ is an autosomal dominant inherited disease which is characterized by left ventricular hypertrophy and clinical manifestations such as congestive heart failure, arrhythmias, and sudden death. Molecu-

Address correspondence to Hideo Fujita, Second Department of Internal Medicine, University of Tokyo, 7-3-1 Hongo, Bunkyo-ku, Tokyo 113, Japan. Phone: 81-3-3815-5411; FAX: 81-3-3814-0021. E-mail: hfujita@medbank.or.jp

Received for publication 14 October 1996 and accepted in revised form 17 December 1996.

1. Abbreviation used in this paper: FHC, familial hypertrophic cardiomyopathy.

J. Clin. Invest.

(C) The American Society for Clinical Investigation, Inc. 0021-9738/97/03/1010/06 \$2.00

Volume 99, Number 5, March 1997, 1010-1015 lar genetic studies have recently revealed that FHC is caused by mutations of genes coding for cardiac sarcomeric proteins, such as myosin heavy chain, troponin T, $\alpha$-tropomyosin, and C-protein (1-8).

FHC is caused by missence mutations in the $\beta$ cardiac myosin heavy chain in 10-30\% of all affected individuals (9). More than 30 such mutations have been reported so far. Most of these mutations are single amino acid replacement in the globular head domain (motor domain) or in the head-rod junction of the molecule. There has been some literature describing individuals with mutations of more conservative amino acid replacement who had a better clinical prognosis (10). These findings suggest that partial loss of myosin motor functions is responsible for the onset of $\mathrm{FHC}$.

To examine the impact of FHC mutations on myosin motor functions, we created myosin mutants equivalent to the FHC mutations, using Dictyostelium discoideum myosin II (designated as myosin) as a model system. Although sequence homology between the motor domain of $D$. discoideum myosin and that of human cardiac $\beta$ myosin is only $40 \%$, their threedimensional structure must be very similar, because crystal structure of the $D$. discoideum myosin motor domain is almost identical to that of chicken skeletal myosin although the two proteins share a $47 \%$ sequence identity (11). Moreover, it was recently proved that other motor proteins, kinesin, and ncd are also similar to myosin in 3-D structure, suggesting the structures may be common among these motor proteins irrespective of their extent of sequence homology $(12,13)$. Therefore the $D$. discoideum myosin mutants can be good models for the FHC mutants as far as mutations in conserved sequences are concerned. Using the $D$. discoideum myosin heavy chain gene, the R397Q, F506C, G575R, A699R, K703Q, and K703W mutants were constructed (Fig. 1); these mutations are in conserved stretches of the sequence and FHC mutations equivalent to them exhibit differences in clinical prognosis among the affected individuals. F506C is equivalent to F513C of FHC (10). This mutation gives a better prognosis among those examined here. The affected family members have a near-normal life expectancy. A699R, K703Q, and K703W are equivalent to G716R, R719Q, and R719W of FHC, respectively (10, 14). These FHC mutations exhibit worse prognosis. The affected families have a high incidence of premature death and an average life expectancy in affected individuals of only $38 \mathrm{yr}$. R397Q and G575R are equivalent to R403Q (2) and G584R of FHC (15), respectively. Level of prognosis of individuals affected by these mutations are that between F513C and those of the G716R/R719Q/R719W group.

Mutant $D$. discoideum myosins equivalent to the FHC mutants were expressed in $D$. discoideum myosin-null cells in which myosin heavy chain gene was disabled by homologous 
recombination $(16,17)$. These mutant myosins were purified, and their motile and enzymatic activities were examined.

\section{Methods}

Vectors and transformation. D. discoideum myosin heavy chain gene and an extrachromosomal expression vector, pBIG, were used to express full-length myosin and its mutants $(18,19)$. Restricted fragments including target sites were subcloned into a plasmid, to which oligonucleotide-mediated mutagenesis was carried out according to the method of Kunkel et al. (20,21). After confirming the mutations, full-length myosin heavy chain genes were reconstructed from mutated fragments. They were then fused to the actin- 15 promoter in frame. Heavy chain genes with the actin promoter were excised and inserted into $\mathrm{pBIG}$. The resulting expression vectors were introduced by electroporation (22) into $D$. discoideum myosin null cells which are incompetent to produce endogenous myosin (16). Transformants were selected by culturing them in the presence of G418.

Expression and purification of myosin. Recombinant myosins were expressed in the transformed cells which were axenically cultured in HL5 medium including G418. Purification of myosins was carried out by modifications of the method previously described (23). All of the following steps were carried out at $0-4^{\circ} \mathrm{C}$. Cells were harvested at saturation $\left(1-2 \times 10^{7}\right.$ cells $\left./ \mathrm{ml}\right)$. They were washed twice with $10 \mathrm{mM}$ Tris $\times \mathrm{HCl}(\mathrm{pH} 7.5)$, and resuspended in lysis buffer consisting of 10 $\mathrm{mM}$ Tris $\times \mathrm{HCl}(\mathrm{pH} 7.5), 40 \mathrm{mM}$ sodium pyrophosphate, $2 \mathrm{mM}$ EDTA, $1 \mathrm{mM}$ DTT with mixture of protease inhibitors such as PMSF, leupeptin, chymostatin, and pepstatin. After adding equal volume of $60 \%$ sucrose in the same buffer, cells were disrupted by sonication for $60 \mathrm{~s}$ on ice. After sonication, $3 \mathrm{M} \mathrm{KCl}$ was added to the disrupted cells to make the final $\mathrm{KCl}$ concentration $0.25 \mathrm{M}$. Then cell debris was sedimented at $10,000 \mathrm{~g}$, and the supernatant was centrifuged again at 541,000 $\mathrm{g}$ in a TL-100 ultracentrifuge (Beckman Instruments, Inc., Fullerton, CA) for $30 \mathrm{~min}$. The clear supernatant was dialyzed overnight against $10 \mathrm{mM}$ Mops $\mathrm{pH} 6.8,50 \mathrm{mM} \mathrm{KCl}, 2 \mathrm{mM}$ EDTA, and $0.5 \mathrm{mM}$ DTT. Actomyosin precipitate was collected by centrifugation at $10,000 \mathrm{~g}$, and resuspended in extraction buffer containing $10 \mathrm{mM}$ Tris $\times \mathrm{HCl}(\mathrm{pH} 7.5), 0.25 \mathrm{M} \mathrm{NaCl}, 7 \mathrm{mM} \mathrm{MgCl}_{2}$, and $10 \mu \mathrm{g} / \mathrm{ml}$ phalloidin. After addition of $5 \mathrm{mM}$ ATP, it was centrifuged at 541,000 $\mathrm{g}$ in TL-100. Six volumes of $40 \mathrm{mM}$ sodium pyrophosphate ( $\mathrm{pH}$ 7.4) were added to the supernatant. It was directly applied to a DEAE-5PW HPLC column (Tosho Inc., Tokyo, Japan). Proteins were eluted by a linear gradient of $0-0.5 \mathrm{M} \mathrm{NaCl}$ in $10 \mathrm{mM}$ Tris $\times \mathrm{HCl}(\mathrm{pH}$ 7.5). Myosin was eluted immediately after a peak of ATP which appeared at $\sim 0.35 \mathrm{M} \mathrm{NaCl}$. Wild type myosin was also purified from AX2 cells in the same way. From $10 \mathrm{~g}$ of wet cells, as much as $300 \mu \mathrm{g}$ of myosin was finally purified from mutant cells.

Force measurements with the laser trap system. Actin was purified from acetone powder of rabbit skeletal muscle as previously described (24). Force generated by a single actin filament and myosin molecules was measured according to the method of Miyata et al. (25). Actin filaments were bound to gelsolin-coated polystyrene beads $(1.0 \mu \mathrm{m}$ diameter; Polysciences Inc., Warrington, PA) by mixing them overnight at $0^{\circ} \mathrm{C}$. Each myosin sample was diluted with a solvent containing $0.6 \mathrm{M} \mathrm{KCl}$, and $50 \mathrm{mM}$ Tris $\times \mathrm{HCl} \mathrm{pH} 7.5$ to a final myosin concentration of $50 \mu \mathrm{g} / \mathrm{ml}$, applied to a nitrocellulosecoated coverslip $(60 \times 30 \mathrm{~mm}$, Matsunami Glass Ind., Ltd., Osaka, Japan), and covered by another smaller coverslip $(18 \times 18 \mathrm{~mm})$. The coverslips were separated by $\sim 100 \mu \mathrm{m}$ with a layer of silicon grease to form a flow cell. After $5 \mathrm{~min}$, BSA $(0.5 \mathrm{mg} / \mathrm{ml})$ was applied to the flow cell to wash out unbound myosin and coat the exposed nitrocellulose surface. Actin filaments attached to beads were suspended in MgATP solution containing $50 \mathrm{mM}$ Imidazole, $25 \mathrm{mM}$ Tris $\times \mathrm{HCl}, 25$ $\mathrm{mM} \mathrm{KCl}, 6 \mathrm{mM} \mathrm{MgCl}_{2}, 1 \mathrm{mM}$ EDTA, and $0.2 \%$ methylcellulose, $\mathrm{pH}$ 7.5 , and introduced to the myosin-coated surface by perfusion. The force measuring system consisted of a fluorescent microscope (Axiovert equipped with an oil immersion objective $100 \times$ NA 1.3 ; Zeiss, Oberkochen, Germany) and a NdYLF laser (OEM 10471000P, wave length 1,047 nm, 1W; Amoco Laser Co., Naperville, IL) coupled with a specially designed optical path apparatus. In each experiment a bead bound to a single actin filament was selected under fluorescent microscope and captured by the laser trap. The position of the bead was detected by a position sensor with a quadratic photodiode

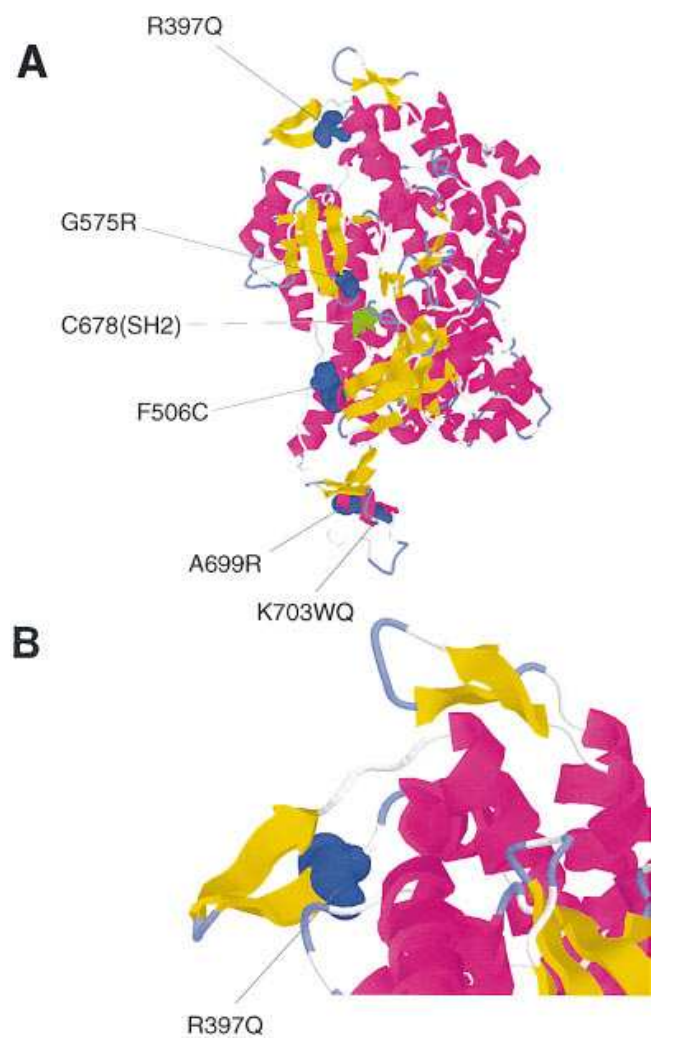

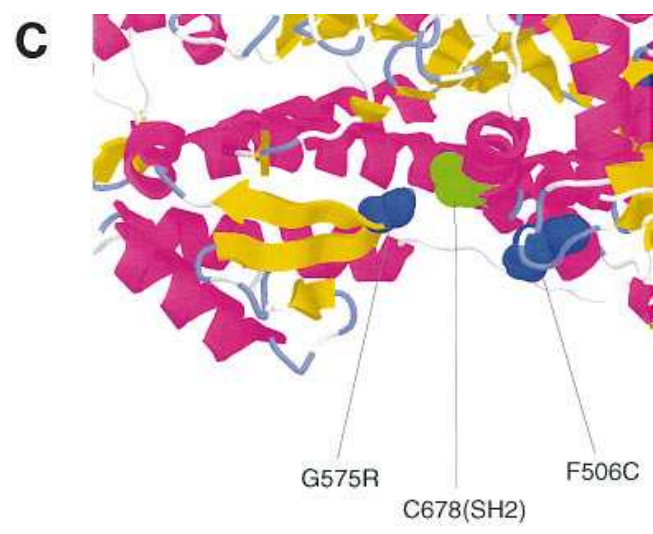

D

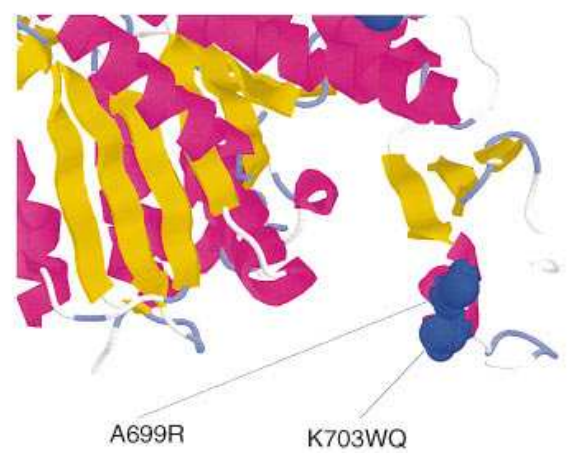

Figure 1. The "motor domain" of $D$. discoideum myosin II S1dC(30) is shown globally in $(A)$, with the mutated amino acid residues indicated. A699 and K703 are on a short $\alpha$-helix in the $\mathrm{COOH}$-terminal subdomain after the $\alpha$-helix corresponding to SH1/SH2. (B) R397 resides at the base of a finger-like $\beta$ structure. (C) G575 resides at a $\beta$ turn close to the $50 \mathrm{~K}$ cleft and also to the reactive cysteine, $\mathrm{SH} 2$ (C678). This figure was drawn with the program RasMol ver. 2.5 (Sayle 1994). 
(model S1557; Hamamatsu Photonics Sys. Corp., Hamamatsu City, Japan) (26). The Hookean constant was found from the fluctuation analysis (27) and ranged form 0.07 to $0.12 \mathrm{pN} / \mathrm{nm}$. The displacement of beads was converted to force by multiplying the Hookean constant.

In vitro motility assay. In vitro motility assay was carried out according to Kron and Spudich with some modifications (28). The assay buffer contained $50 \mathrm{mM}$ Imidazole ( $\mathrm{pH} 7.5), 25 \mathrm{mM} \mathrm{KCl}, 6 \mathrm{mM}$ $\mathrm{MgCl}_{2}, 0.1 \mathrm{mM}$ EGTA, $1 \mathrm{mM}$ ATP, $1 \mathrm{mM}$ DTT, $2.5 \mathrm{mg} / \mathrm{ml}$ glucose, $0.1 \mathrm{mg} / \mathrm{ml}$ glucose oxidase, and $0.02 \mathrm{mg} / \mathrm{ml}$ catalase with 0.2 or $0.7 \%$ methylcellulose. The assays were performed at $25^{\circ} \mathrm{C}$ with the same apparatus for force-measurement as described above.

Measurements of actin-actived $\mathrm{Mg}^{2+}$ ATPase activity. Malachite green method was used to measure the actin-activated $\mathrm{Mg}^{2+}$ ATPase activity (29). The assay buffer contained $18 \mathrm{mM}$ Mops, $\mathrm{pH}$ 7.4, 12.5 $\mathrm{mM} \mathrm{KCl}, 4 \mathrm{mM} \mathrm{MgCl}, 1 \mathrm{mM}$ ATP, and $0.1 \mathrm{mM}$ DTT. ATPase assay was performed at $25^{\circ} \mathrm{C}$. Data were obtained at more than eight different concentrations of actin. Each measurement was carried out in duplicate. $V_{\max }$ and $K_{\mathrm{m}}$ were obtained from double reciprocal plot of actin concentration $([\mathrm{S}])$ and hydrolysis rate $(\mathrm{v})$.

Prediction of structures of mutant myosins. Based on the crystal structure of the motor domain of $D$. discoideum myosin II $(11,30)$, structures of mutant myosins were calculated by homology modeling, using the CARA/SegMod program (Molecular Application Group, Palo Alto, CA) (31).

\section{Results}

By comparing amino acid sequences of corresponding regions of human cardiac $\beta$ myosin heavy chain (Fig. $2 A$ ) and $D$. discoideum myosin heavy chain (Fig. 2 B) FHC-like mutations, R397Q, F506C, G575R, A699R, K703Q, and K703W, were selected for introduction into the $D$. discoideum myosin heavy chain (Fig. 2, bold letters). These $D$. discoideum myosin mutants equivalent to the FHC mutants were expressed in $D$. discoideum myosin-null cells $(18,19)$. As a control, the wild type myosin was also expressed in myosin-null cells. Expression levels of the wild type and mutant myosin heavy chains were similar to that of the endogenous myosin heavy chain in AX2 cells. The wild type and mutant myosins were purified from the transformed myosin-null cells (see Methods). Yields and purities of these myosins were very similar to those of the wild type myosin purified from AX2 cells. Compared with the wild type myosin isolated from AX2 cells, these myosins contained normal amount of essential and regulatory light chains (Fig. 3).

Enzymatic and motile activities of the wild type and mutant myosins were examined. Compared with the wild type, all mutant myosins examined produced less force with reduced actinactivated ATPase activity and reduced velocity of actin filaments (Table I, Figs. 4 and 5). The data enabled us to group these mutants into three classes by their functional properties as well as to the clinical prognoses accompanying the corresponding FHC mutations. The first class consists of three mutants, A699R, K703Q, and K703W. The corresponding FHC mutations, G716R, R719Q, and R719W, exhibit the worst prognosis among those studied here. The second class consists of only one mutant F506C. The equivalent FHC mutation F513C gives a fairly good prognosis. The third class consists of R397Q and G575R. The corresponding FHC mutations are $\mathrm{R} 403 \mathrm{Q}$ and G584R. The prognosis of affected individuals is worse than that of the second class, but better than that of the first class.

The first mutations, A699R, K703Q, and K703W, entail charge changes. Both A699 and K703 are on a short $\alpha$-helix in
A

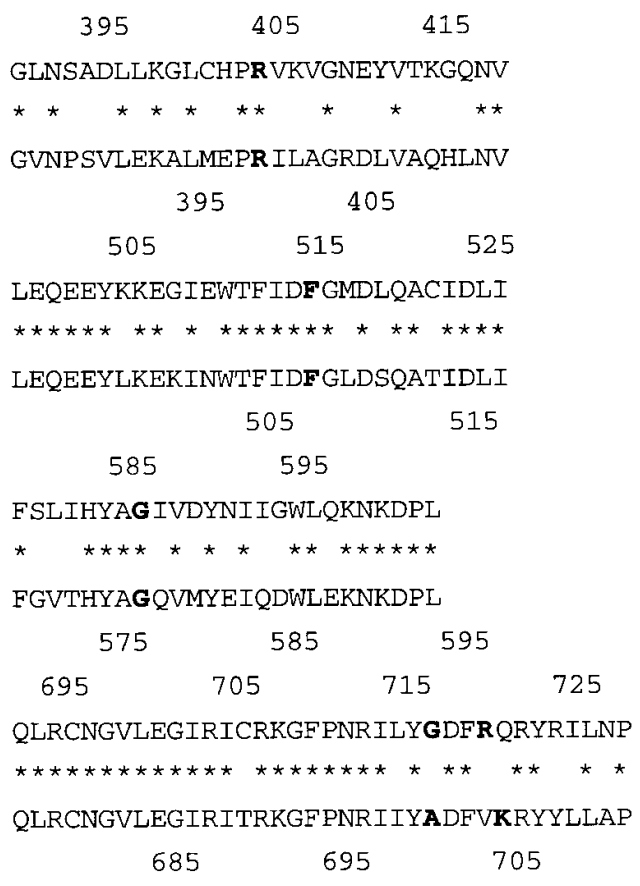

Figure 2. Design of mutant myosins. The mutated amino acid residues in FHC and the human cardiac sequences around them are shown in the upper row. Corresponding D. discoideum sequences are shown in the lower row. * Indicates homologous amino acid residues. Bold letters indicate the mutated residues. $(A) \mathrm{R} 397,(B) \mathrm{F} 506,(C)$ G575, and (D) A699 and K703.

the $\mathrm{COOH}$-terminal subdomain after the $\alpha$-helix (Fig. 1) corresponding to $\mathrm{SH} 1 / \mathrm{SH} 2$ (30). This class of mutant myosins showed functional properties distinct from others. Compared with the wild type, $K_{\mathrm{m}}$ values of the actin-activated ATPase activity increased dramatically. They were $20.2 \times \mathrm{WT}$ (WT is the corresponding value of the wild type myosin), $6.4 \times \mathrm{WT}$ and $11.4 \times$ WT for A699R, K703Q, and K703W, respectively (Table I). Contrary to $K_{\mathrm{m}}, V_{\max }$ values were only moderately re-

Table I. Summary of Actin-activated $\mathrm{Mg}^{2+}$ ATPase Activities

\begin{tabular}{llllc}
\hline & $V_{\max }$ & Ratio & $K_{\mathrm{m}}$ & Ratio \\
\hline & $s^{-1}$ & & $\mu M$ & \\
AX2 & 2.9 & & 0.38 & \\
Recombinant & & & & \\
$\quad$ wild type & 2.7 & 1 & 0.45 & 1 \\
R397Q & 0.88 & 0.33 & 0.99 & 2.2 \\
F506C & 1.18 & 0.44 & 0.09 & 0.2 \\
G575R & 0.68 & 0.25 & 1.08 & 2.4 \\
A699R & 1.58 & 0.59 & 9.08 & 20 \\
K703Q & 1.07 & 0.4 & 2.87 & 6.4 \\
K703W & 1.92 & 0.71 & 5.13 & 11 \\
& & & & \\
\hline
\end{tabular}

The $\mathrm{V}_{\max }$ value was decreased for all mutants. The $K_{\mathrm{m}}$ values of A699R, $\mathrm{K} 703 \mathrm{Q}$, and $\mathrm{K} 703 \mathrm{~W}$ were much more elevated than those of the others. Data were obtained at more than eight different concentrations of actin. $V_{\max }$ and $K_{\mathrm{m}}$ were determined from double reciprocal plots of actin concentration and hydrolysis rate. The ratios compared with the recombinant wild type are also indicated in the table. 
AX2 WT R397Q F506C G575R A699R K703Q K703W

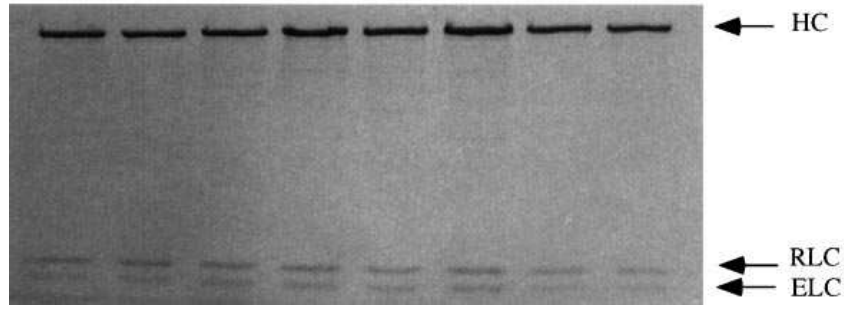

Figure 3. $12.5 \%$ SDS-polyacrylamide gel electrophoresis of the purified myosins. In the mutant myosins, the amount of the heavy and light chains is very similar to those in the wild type. The gel patterns also show that the purified myosins are completely free of actin.

duced. They were $0.59 \times \mathrm{WT}, 0.40 \times \mathrm{WT}, 0.71 \times \mathrm{WT}$ for A699R, K703Q, and K703W, respectively. These high $K_{\mathrm{m}}$ values imply very weak interactions of these mutant myosins with actin filaments. The notion agrees well with the finding that sliding velocity of actin filaments on these mutant myosins was strongly dependent on methylcellulose concentrations (Fig. 4). Consistent with moderately reduced $V_{\max }$ values $(0.40-0.71 \times$ WT), filaments slid on these mutant myosins at moderately lower velocity than on the wild type myosin at $0.7 \%$ methylcellulose. They were $0.41 \times \mathrm{WT}, 0.68 \times \mathrm{WT}$, and $0.33 \times \mathrm{WT}$ for A699R, K703Q and K703W, respectively. At 0.2\% methylcellulose, however, only marginal sliding was observed. Sliding velocities of actin filaments on the mutant myosins were $0.11 \times$ WT, $0.19 \times \mathrm{WT}$, and $0.06 \times \mathrm{WT}$ for A699R, K703Q, and $\mathrm{K} 703 \mathrm{~W}$, respectively. It seems that due to weak interactions between mutant myosin heads and actin filaments, effective number of myosins interacting with an actin filament was not large enough to attain the saturating level of velocity of the actin filament even in the presence of methylcellulose (32). By increasing concentration of methylcellulose from $0.2 \%$ to $0.7 \%$, actin filaments would be forced to stay on the myosincoated surface longer, resulting in increase of number of myo-

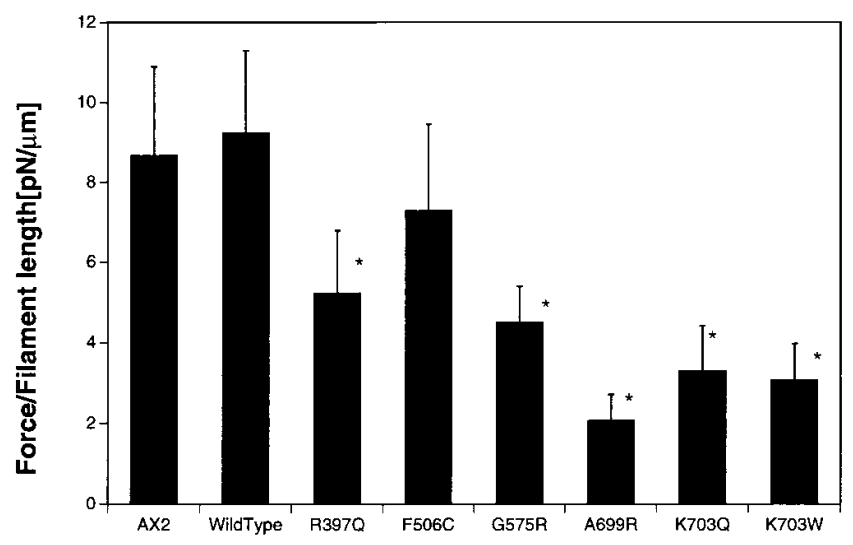

Figure 4. The force generated by a single actin filament and mutant myosins. There are marked differences in the forces generated by the mutant myosins. The force of F506C was as high as that of the wild type. In contrast, the force generated by G575R, A699R, K703Q, and K703W was relatively low among the mutants. The data are expressed as mean \pm SD (bars) of the ratio of Force/Filament length $[\mathrm{pN} / \mu \mathrm{m}] . * P<0.001$ vs. wild type. sin heads effectively interacting with actin filaments, and then in increase of the velocity as observed here. Thus the observed dependence of sliding velocity could indicate that interactions between actin filaments and myosin head are very weak during the sliding motion, and it is possible that these velocities in $0.7 \%$ methylcellulose did not reach the maximum. As expected from their enzymatic and motile properties, force generated by these mutant myosins was distinctively low; $0.22 \times$ $\mathrm{WT}, 0.36 \times \mathrm{WT}$, and $0.33 \times \mathrm{WT}$ for $\mathrm{A} 699 \mathrm{R}, \mathrm{K} 703 \mathrm{Q}$, and $\mathrm{K} 703 \mathrm{~W}$, respectively.

The second class of mutants, F506C, accompanies no charge changes. F506 is on a long $\alpha$-helix in the lower part of the motor domain located away from the actin-binding site and the ATPase pocket (Fig. 1) (11,30). Replacement of this residue to another hydrophobic residue resulted in moderate changes of its enzymatic properties. By this mutation, $V_{\max }$ value decreased to $0.44 \times \mathrm{WT}$, and $K_{\mathrm{m}}$ value also decreased to $0.20 \times \mathrm{WT}$ as shown in Table I. As shown in Fig. 4, sliding velocity of actin filaments on this mutant myosin remains higher $(0.67 \times$ WT $)$ than those of other types of mutants irrespective of methylcellulose concentrations. Force level of this mutant was nearly normal as shown in Fig. $5(0.79 \times$ WT $)$.

The third class of mutants, R397Q and G575R, is accompanied by charge changes to the opposite direction, i.e., increase or decrease of one positive charge. R397 resides at the base of a finger-like $\beta$ structure protruding out from the upper $50-\mathrm{K}$ subdomain, which possibly interacts with actin during force generation (33). The R397Q mutant myosin showed a distinct profile of actin-activated ATPase activity: $V_{\max }$ value dropped to $0.33 \times$ WT while its $K_{\mathrm{m}}$ value increased to $2.2 \times$ WT (Table I). The sliding velocity of actin filaments depends on methylcellulose concentrations, though not as dramatic as the first class of mutants. Increase of the concentration from 0.2 to $0.7 \%$ resulted in increase of the sliding velocity from $0.50 \times$ WT to $0.74 \times \mathrm{WT}$, an indication that the actin-myosin interaction was weak during the sliding motion. This result is consis-

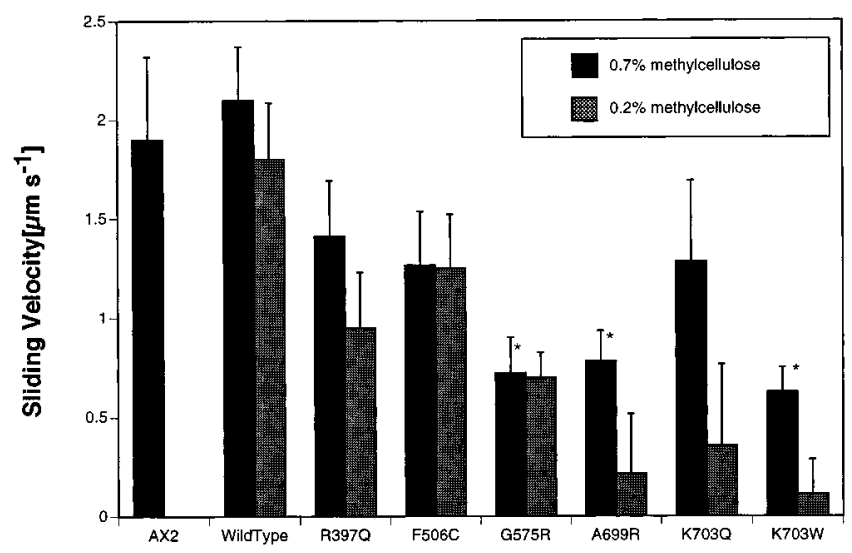

Figure 5. Sliding velocity of actin filaments. F506C and R397Q gave relatively high sliding velocity of actin. Marked elevation of sliding velocity with a higher concentration $(0.7 \%)$ of methylcellulose was observed for mutants A699R, K703Q, and K703W. The data are expressed as mean $\pm \mathrm{SD}$ (bars) for $30-50$ actin filaments in a single assay. Filled bars represent the sliding velocity in assay buffer containing $0.7 \%$ methylcellulose. Shaded bars show the velocity in the presence of $0.2 \%$ methylcellulose. $* P<0.001$ vs. wild type (with $0.7 \%$ methylcellulose). 
tent with previous reports that the corresponding R403Q mutation in rat $\alpha$ cardiac myosin heavy chain resulted in weaker actin-myosin interaction (34). Force level generated by the $\mathrm{R} 397 \mathrm{Q}$ myosin $(0.57 \times \mathrm{WT})$ was higher than that of the first class but lower than that of the second class.

G575 resides at a $\beta$ turn in the lower $50-\mathrm{K}$ subdomain and close to the reactive $\mathrm{SH} 2$ cysteine (Fig. 1). G575R mutation again resulted in similar changes in the actin-activated ATPase activity, i.e., appreciable decrease of $V_{\max }$ value $(0.25 \times \mathrm{WT})$ and moderate increase of $K_{\mathrm{m}}$ value $(2.4 \times \mathrm{WT})$. Consistent with the lower $V_{\max }$ value, sliding velocity of actin filaments on the G575R myosin molecules was slower than that of the wild type. However, the velocity did not much depend on methylcellulose concentration; $0.37 \times \mathrm{WT}$ and $0.38 \times \mathrm{WT}$ at $0.2 \%$ and $0.7 \%$, respectively. Force level of this mutant myosin was similar to that of the R397Q myosin $(0.49 \times \mathrm{WT})$.

\section{Discussion}

The first class of D. discoideum mutants, A699R, K703Q, and $\mathrm{K} 703 \mathrm{~W}$, is characterized by their dramatically high $K_{\mathrm{m}}$, only moderately reduced $V_{\max }$ and low level of force. In the $D$. discoideum motor domain, A699 and K703 are on an $\alpha$-helix in the $\mathrm{COOH}$-terminal subdomain after the conservative SH1/ $\mathrm{SH} 2$-corresponding helix. This $\mathrm{COOH}$-terminal subdomain rich in $\alpha$ helices and $\beta$ sheets is located at the distal end of the motor domain away from the actin-binding sites. Therefore, it is very unexpected that A699R, K703Q, and K703W mutations affect the actin-myosin interaction. Since the A699R, K703Q, and $\mathrm{K} 703 \mathrm{~W}$ mutations would induce only minor distortion of the $\alpha$ helix as suggested by the model building calculations (CARA/SegMod program; Molecular Application Groups; data not shown), they would mainly change interactions between the $\mathrm{COOH}$-terminal subdomain with another subdomain of myosin. It has been recently shown that the $\mathrm{COOH}-$ terminal subdomain can take two different orientations against the remaining part of the motor domain, depending on states of nucleotide at the ATPase pocket (30). The COOH-terminal subdomain may swing from one orientation to the other during the cyclic ATP hydrolysis, amplifying the movements of myosin motor domain $(35,36)$. These considerations lead us to speculate that mutations at the $\mathrm{COOH}$-terminal domain might reduce the affinity with actin filaments by affecting this swing motion, because the A699R, K703Q, and K703W mutations would alter ionic and hydrophobic properties of the surface of this subdomain. It must be mentioned here that the mutated residues are close to the essential light chain which binds to a long $\alpha$-helix after the COOH-terminal subdomain $(11,33,37)$. It seems that dramatically weakened interactions of this class of mutants with actin filaments caused by the impaired essential light chain (ELC) interface would lead to the lowest level of force among those examined here, and to the worst prognosis of the corresponding FHC. Poetter et al. recently reported that the FHC R719 mutation of human $\beta$ myosin increased the sliding velocity of actin filaments, implying weaker interactions of actin and the mutant myosins $(38,39)$. Another FHC mutation in essential light chain (M149V) also resulted in higher velocity of actin filaments, implying weaker interactions of actin and the mutant myosins. The results are partially consistent with our data because they suggest that the weaker ac- tin-myosin interactions are reflected directly in force level generated by these $D$. discoideum mutant myosins.

Moderately reduced $V_{\max }$ and $K_{\mathrm{m}}$, and nearly normal motile properties are the characteristics of the second one, F506C. F506 is located on a long a-helix away from the actin-binding site and the ATPase pocket $(11,30)$. This helix is composed of the longest sequence-conserved stretch of myosin, and is connected to the ATPase site through a loop containing the pivotal Gly457, which may play an essential role as a hinge to open and close the 50-K cleft (11). Replacement of F506 with another hydrophobic residue would not disrupt the helix, though side chain rearrangements would take place as suggested by the structure prediction of the mutant myosin (data not shown). The notion is also consistent with our observation that the F506C mutation in D. discoideum myosin showed nearly normal motor functions. Nearly normal motor functions, especially nearly normal force level, of the $D$. discoideum F506C mutant is in concert with the better clinical prognosis of individuals affected by the corresponding FHC mutation.

The third class, R397Q and G575R, are characterized by low $V_{\text {max }}$, moderately increased $K_{\mathrm{m}}$ and moderately reduced force level. R397 resides at the base of a finger-like structure composed of two $\beta$ strands, which is located at the tip of the motor domain and would be a part of the actin-binding site on myo$\sin$ (33). Because the finger-like structure is stabilized by strong hydrophobic interactions, the R397Q mutation would scarcely affect the structure as suggested by the model building calculation (data not shown). Thus it is likely that the R397Q mutation would affect the ionic interaction between actin and myosin during force generation. Because positive charges on the myosin surface interact with acidic residues at the $\mathrm{NH}_{2}$ terminus and on the loop containing D24 and D25 (40-44), loss of one positive charge at the actin-binding surface would weaken the actin-myosin interaction. The weak actin-myosin interaction of the corresponding FHC mutant would then result in lower level of force, leading to the onset of FHC.

G575 is located closely to the $50-\mathrm{K}$ cleft and at a turn connecting two $\beta$ strands. Replacement of the glycine residue with the bulky arginine residue can be accommodated into the original structure without much distortion of the peptide folding though side chain rearrangements may take place, as suggested by the model building (data not shown). The turn is in contact with the long $\alpha$-helix connected to the ATPase site through a loop where the pivotal G457 resides. Introduction of a positive charge to the contact site would affect the helix rearrangement during ATP hydrolysis. This might indirectly reduce the apparent affinity of the mutant myosin with actin filaments, and then its force level.

Comparison of the enzymatic and motile properties of these $D$. discoideum mutants with clinical prognosis of individuals affected by the corresponding FHC mutants leads us to conclude that the force produced by mutant myosins depends on their affinity to actin filaments during ATP hydrolysis in Dictyostelium myosin, and to speculate that the heavy chainessential light chain interface plays an important role in production of force.

Though there may be some limitations for relating crossspecies results to human disease, our data suggest that molecular-basis force of myosin mutants may be one of the important primary factors of pathogenesis which affect the prognosis of human FHC. This hypothesis is to be further investigated. 


\section{Acknowledgments}

We thank Ms. Reiko Okura for her excellent technical assistance. The coordinates of the motor domain of Dictyostelium myosin II was kindly supplied by Dr. Ivan Rayment (University of Wisconsin, Madison, WI). The myosin-null cell and the pBIG vector were kindly supplied by Drs. James A. Spudich (Stanford University, Palo Alto, CA) and Bruce Patterson (University of Arizona, Tucson, AZ). Structure calculations by the CARA/SedMod program were carried out with the help of the Ryoka System Co. Ltd., Tokyo, Japan.

This work was supported by Grants-in-Aid from the Ministry of Education, Science and Culture of Japan to K. Sutoh and S. Sugiura. Financial support from the Mitsubishi Foundation and the Uehara Foundation to K. Sutoh is gratefully acknowledged.

\section{References}

1. Tanigawa, G., J.A. Jarcho, S. Kass, S.D. Solomon, H.P. Vosberg, J.G. Seidman, and C.E. Seidman. 1990. A molecular basis for familial hypertrophic cardiomyopathy: an alpha/beta cardiac myosin heavy chain hybrid gene. Cell. 62:991-998.

2. Geisterfer, L.A., S. Kass, G. Tanigawa, H.P. Vosberg, W. McKenna, C.E. Seidman, and J.G. Seidman. 1990. A molecular basis for familial hypertrophic cardiomyopathy: a beta cardiac myosin heavy chain gene missense mutation. Cell. 62:999-1006.

3. Seidman, C.E., and J.G. Seidman. 1991. Mutations in cardiac myosin heavy chain genes cause familial hypertrophic cardiomyopathy. Mol. Biol. Med. $8: 159-166$.

4. Thierfelder, L., H. Watkins, C. MacRae, R. Lamas, W. McKenna, H.P. Vosberg, J.G. Seidman, and C.E. Seidman. 1994. Alpha-tropomyosin and cardiac troponin $\mathrm{T}$ mutations cause familial hypertrophic cardiomyopathy: a disease of the sarcomere. Cell. 77:701-712.

5. Watkins, H., D. Conner, L. Thierfelder, J.A. Jarcho, C. MacRae, W.J. McKenna, B.J. Maron, J.G. Seidman, and C.E. Seidman. 1995. Mutations in the cardiac myosin binding protein-C gene on chromosome 11 cause familial hypertrophic cardiomyopathy. Nat. Genet. 11:434-437.

6. Watkins, H., W.J. McKenna, L. Thierfelder, H.J. Suk, R. Anan, A. O'Donoghue, P. Spirito, A. Matsumori, C.S. Moravec, J.G. Seidman, et al. 1995. Mutations in the genes for cardiac troponin T and alpha-tropomyosin in hypertrophic cardiomyopathy. N. Engl. J. Med. 332:1058-1064.

7. Watkins, H., C. MacRae, L. Thierfelder, Y.H. Chou, M. Frenneaux, W. McKenna, J.G. Seidman, and C.E. Seidman. 1993. A disease locus for familial hypertrophic cardiomyopathy maps to chromosome 1q3. Nat. Genet. 3:333-337.

8. Bonne, G., L. Carrier, J. Bercovici, C. Cruaud, P. Richard, B. Hainque, M. Gautel, S. Labeit, M. James, J. Beckmann, et al. 1995. Cardiac myosin binding protein-C gene splice acceptor site mutation is associated with familial hypertrophic cardiomyopathy. Nat Genet. 11:438-440.

9. Rayment, I., H.M. Holden, J.R. Sellers, L. Fananapazir, and N.D. Epstein. 1995. Structural interpretation of the mutations in the beta-cardiac myosin that have been implicated in familial hypertrophic cardiomyopathy. Proc. Natl. Acad. Sci. USA. 92:3864-3868.

10. Anan, R., G. Greve, L. Thierfelder, H. Watkins, W.J. McKenna, S. Solomon, C. Vecchio, H. Shono, S. Nakao, H. Tanaka, et al. 1994. Prognostic implications of novel beta cardiac myosin heavy chain gene mutations that cause familial hypertrophic cardiomyopathy [see comments]. J. Clin. Invest. 93:280285.

11. Fisher, A.J., C.A. Smith, J.B. Thoden, R. Smith, K. Sutoh, H.M. Holden, and I. Rayment. 1995. X-ray structures of the myosin motor domain of dictyostelium discoideum complexed with $\mathrm{MgADP} \mathrm{BeFx}$ and $\mathrm{MgADP} \mathrm{A}_{1} \mathrm{~F}_{4}^{-}$. Biochemistry. 34:8960-8972.

12. Sablin, E.P., F.J. Kull, R. Cooke, R.D. Vale, and R.J. Fletterick. 1996. Crystal structure of the motor domain of the kinesin-related motor ncd. Nature (Lond.). 380:555-559.

13. Kull, F.J., E.P. Sablin, R. Lau, R.J. Fletterick, and R.D. Vale. 1996. Crystal structure of the kinesin motor domain reveals a structural similarity to myosin. Nature (Lond.). 380:550-555.

14. Consevage, M.W., G.C. Salada, B.G. Baylen, R.L. Ladda, and P.K. Rogan. 1994. A new missense mutation, Arg719Gln, in the beta-cardiac heavy chain myosin gene of patients with familial hypertrophic cardiomyopathy. Hum. Mol. Genet. 3:1025-1026.

15. Watkins, H., L. Thierfelder, R. Anan, J. Jarcho, A. Matsumori, W. McKenna, J.G. Seidman, and C.E. Seidman. 1993. Independent origin of identical beta cardiac myosin heavy-chain mutations in hypertrophic cardiomyopathy. Am. J. Hum. Genet. 53:1180-1185.

16. Manstein, D.J., M.A. Titus, A. De Lozanne, and J.A. Spudich. 1989. Gene replacement in Dictyostelium: generation of myosin null mutants. $E M B O$ (Eur. Mol. Biol. Organ.) J. 8:923-932.
17. Manstein, D.J., K.M. Ruppel, and J.A. Spudich. 1989. Expression and characterization of a functional myosin head fragment in Dictyostelium discoideum. Science (USA). 246:656-658.

18. Uyeda, T.Q., and J.A. Spudich. 1993. A functional recombinant myosin II lacking a regulatory light chain-binding site. Science (USA). 262:1867-1870.

19. Ruppel, K.M., T.Q. Uyeda, and J.A. Spudich. 1994. Role of highly conserved lysine 130 of myosin motor domain. In vivo and in vitro characterization of site specifically mutated myosin. J. Biol. Chem. 269:18773-18780.

20. Kunkel, T.A. 1985. Rapid and efficient site-specific mutagenesis without phenotypic selection. Proc. Natl. Acad. Sci. USA. 82:488-492.

21. Kunkel, T.A., J.D. Roberts, and R.A. Zakour. 1987. Rapid and efficient site-specific mutagenesis without phenotypic selection. Methods Enzymol. 154: 367-382.

22. Howard, P.K., K.G. Ahern, and R.A. Firtel. 1988. Establishment of a transient expression system for Dictyostelium discoideum. Nucleic Acids. Res. 16:2613-2623.

23. Ruppel, K.M., T.T. Egelhoff, and J.A. Spudich. 1990. Purification of a functional recombinant myosin fragment from Dictyostelium discoideum. Ann. NY Acad. Sci. 84:147-155.

24. Spudich, J.A., and S. Watt. 1971. The regulation of rabbit skeletal muscle contraction. I. Biochemical studies of the interaction of the tropomyosintroponin complex with actin and the proteolytic fragments of myosin. J. Biol. Chem. 246:4866-4871.

25. Miyata, H., H. Hakozaki, H. Yoshikawa, N. Suzuki, K. Kinoshita, T. Nishizawa, and S. Ishiwata. 1994. Stepwise motion of an actin filament over a small number of heavy meromyosin molecules is revealed in an in vitro motility assay. J. Biochem. 115:644-647.

26. Kamimura, S. 1987. Direct measurement of nanometric displacement under an optical microscope. Appl. Opt. 26:3425-3434.

27. Svoboda, K., and S.M. Block. 1994. Fluctuation analysis of motor protein movement and single enzyme kinetics. Proc. Natl. Acad. Sci. USA. 91: 11782-11786.

28. Kron, S.J., and J.A. Spudich. 1986. Fluorescent actin filaments move on myosin fixed to a glass surface. Proc. Natl. Acad. Sci. USA. 83:6272-6276.

29. Kodama, T., K. Fukui, and K. Kometani. 1986. The initial phosphate burst in ATP hydrolysis by myosin and subfragment-1 as studied by a modified malachite green method for determination of inorganic phosphate. J. Biochem. (Tokyo). 99:1465-1472.

30. Smith, C.A., and I. Rayment. 1996. X-ray structure of the magnesium(II).ADP.vanadate complex of the Dictyostelium discoideum myosin motor domain to 1.9 A resolution. Biochemistry. 35:5404-5417.

31. Lee, C. 1994. Predicting protein mutant energetics by self-consistent ensemble optimization. J. Mol. Biol. 236:918-939.

32. Uyeda, T.Q., S.J. Kron, and J.A. Spudich. 1990. Myosin step size. Estimation from slow sliding movement of actin over low densities of heavy meromyosin. J. Mol. Biol. 214:699-710.

33. Milligan, R.A. 1996. Protein-protein interactions in the rigor actomyosin complex. Proc. Natl. Acad. Sci. USA. 93:21-26.

34. Sweeney, H.L., A.J. Straceski, L.A. Leinwand, B.A. Tikunov, and L. Faust. 1994. Heterologous expression of a cardiomyopathic myosin that is defective in its actin interaction. J. Biol. Chem. 269:1603-1606.

35. Whittaker, M., E.M. Wilson-Kubalek, J.E. Smith, L. Faust, R.A. Milligan, and H.L. Sweeney. 1995. A 35-Å movement of smooth muscle myosin on ADP release. Nature (Lond.). 378:748-753.

36. Uyeda, T.Q., P.D. Abramson, and J.A. Spudich. 1996. The neck region of the myosin motor domain acts as a lever arm to generate movement. Proc. Natl. Acad. Sci. USA. 93:4459-4464.

37. Rayment, I., H.M. Holden, M. Whittaker, C. Yohn, B.M. Lorenz, K.C. Holes, and R.A. Milligan. 1993. Three-dimensional structure of myosin subfragment-1: a molecular motor. Science (Wash. DC). 261:50-58.

38. Poetter, K., H. Jiang, S. Hassanzadeh, S.R. Master, A. Chang, M.C. Dalakas, I. Rayment, J.R. Sellers, L. Fananapazir, and N.D. Epstein. 1996. Mutations in either the essential or regulatory light chains of myosin are associated with a rare myopathy in human heart and skeletal muscle. Nat. Genet. 13:63-69.

39. Lankford, E.B., N.D. Epstein, L. Fananapazir, and H.L. Sweeney. 1995. Abnormal contractile properties of muscle fibers expressing beta-myosin heavy chain gene mutations in patients with hypertrophic cardiomyopathy. J. Clin. Invest. 95:1409-1414.

40. Sutoh, K., M. Andou, K. Sutoh, and Y.Y. Toyoshima. 1991. Site-directed mutations of Dictyostelium actin: Disruption of a negative charge cluster at the N terminus. Proc. Natl. Acad. Sci. USA. 88:7711-7714.

41. Johara, M., Y.Y. Toyoshima, A. Ishijima, H. Kojima, T. Yanagida, and K. Sutoh. 1993. Charge-reversion mutagenesis of Dictyostelium actin to map the surface recognized by myosin during ATP-driven sliding motion. Proc. Natl. Acad. Sci. USA. 90:2127-2131.

42. Duong, A.M., and E. Reisler. 1994. C-terminus on actin: spectroscopic and immunochemical examination of its role in actomyosin interactions. Adv. Exp. Med. Biol. 358:59-70.

43. Miller, C.J., and E. Reisler. 1995. Role of charged amino acid pairs in subdomain-1 of actin in interactions with myosin. Biochemistry. 34:2694-2700.

44. Miller, C.J., P. Cheung, P. White, and E. Reisler. 1995. Actin's view of actomyosin interface. Biophys. J. 68:50S-54S. 